\title{
Radiation-Affected Laminar Flame Propagation
}

\author{
VEDAT S. ARPACI \\ Department of Mechanical Engineering \& Applied Mechanics, The University of Michigan, Ann Arbor, Michigan 48109 \\ and
}

RODNEY J. TABACZYNSKI

Engineering Research Staff, Ford Motor Company, Dearborn, Michigan 4812I

\begin{abstract}
Increased laminar flame thickness and flame speed under the influence of radiation is shown in terms of an original heat transfer number

$H=\frac{\eta \tau P}{1+3 \tau^{2} /(1-\omega)} \sim \frac{\text { Total radiation }}{\text { Conduction }}$

where $\eta=\left(\kappa_{\mathrm{p}} / \kappa_{\mathrm{R}}\right)^{1 / 2}$ is the weighted nongreyness, $\kappa_{\mathrm{p}}$ and $\kappa_{\mathrm{R}}$ are the Planck mean and the Rosseland mean of the absorption coefficient, $\tau=\kappa_{\mathrm{M}} \delta_{\mathrm{K}}$ is optical thickness, $\kappa_{\mathrm{M}}=\left(\kappa_{\mathrm{p}} \kappa_{\mathrm{R}}\right)^{1 / 2}$ the mean absorption, $\delta_{\mathrm{K}}$ the conduction flame thickness, $P=4 \sigma T_{\mathrm{M}}^{3} /\left(\lambda / \delta_{\mathrm{K}}\right)$ the Planck number, $T_{\mathrm{M}}$ the adiabatic flame temperature, $\lambda$ the thermal conductivity, and $\omega$ the albedo, the ratio of scattering to extinction.
\end{abstract}

\section{INTRODUCTION}

Although the importance of radiation on the ignition of pulverized coal was recognized by Nusselt [1] more than five decades ago, later develop. ments were carried out by few pioneers, notably by Hottel, and the results have been utilized by few designers of furnaces. Only during the past three decades has the effect of gas radiation on many technological and environmental problems became widely recognized. More specifically, the radiation in flames, fires, smoke, combustors, diesel chambers, biosphere, as well as in furnaces, has begun to receive increased attention. Because of the quantity of the literature, no attempt is made here to give a complete list of references. Only the latest review articles, or those of a special nature, are mentioned. Recent reviews of the radiation ef- fects in coal combustion may be found in Refs. [2-6]. Also, the influence of alternative fuels on the radiation in combustion chambers is included in Ref. [2]. Fire radiation is reviewed by de Ris [7]. Infrared flame radiation is studied by Buckius and Tien [8]. Recent developments on the zonal and flux methods are compared by Selcuk, Siddall, and Beér [9].

In spite of the extensive efforts summarized above, and perhaps because of the urgent need for specific answers to a variety of technological problems, some fundamental concepts associated with radiation apparently have not been treated or have been given insufficient attention. For example, a dimensionless number which clearly shows the emission, absorption, and scattering characteristics of radiation appears to be unknown. This dimensionless number is essential for a fundamental understanding of radiation effects. The 
major objective of this study is to introduce this number, and understand in terms of this number the qualitative effects of gas radiation on laminar flame propagation. Monochromatic behavior and spectral effects of radiation, although important for quantitative studies, are not considered here. The outline of the work is as follows: In Section 2, the foundations of gas radiation are reviewed, and some qualitative conclusions are drawn on dimensional grounds. In Section 3, the effect of radiation on the laminar flame propagation is discussed following the qualitative thermal theory of Landau [10] (see also Williams [11] or Glassman [12]), to be extended by the addition of the radiation effect. In Section 4, after a discussion of the dimensionless numbers involved, some remarks are made about the quantitative effects of radiation on the laminar flame propagation.

\section{GAS RADIATION}

As is well known, the major contributions of thermal radiation to heat transfer problems is the radiation heat flux $q_{i}{ }^{\mathbf{R}}$ (see for example, Sparrow and Cess [13], Vincenti and Kruger [14], Sampson [15], Ozisik [16], or Howell and Siegel [17]). For the thin gas limit and the thick gas limit, we have for this flux

$\frac{\partial q_{i}^{\mathrm{R}}}{\partial x_{i}}=-4 \kappa_{\mathrm{P}} E_{b}$,

$q_{i}{ }^{\mathbf{R}}=-\frac{4}{3 \beta_{\mathrm{R}}} \frac{\partial E_{\mathrm{b}}}{\partial x_{i}}$,

where $E_{\mathrm{b}}$ is the blackbody emissive power, $\kappa_{\mathrm{P}}$ is the Planck mean of the absorption coefficient, and $\beta_{R}$ is the Rosseland mean of the extinction coefficient. Now introduce $\beta_{R}=\kappa_{R}+\sigma_{R}$, where $\kappa_{R}$ and $\sigma_{R}$ are the Rosseland mean of the absorption and scattering coefficients, the albedo $\omega=\sigma_{R} / \beta_{R}$, the mean absorption coefficient $\kappa_{M}=\left(\kappa_{P} \kappa_{R}\right)^{1 / 2}$, the weighted nongreyness $\eta=\left(\kappa_{P} / \kappa_{R}\right)^{1 / 2}$, and the optical thickness $\tau=\kappa_{\mathrm{M}} l, l$ being a characteristic length to be commented on later. Then Eqs. (1) and (2) may be replaced, on dimensional grounds, with

$q^{\mathbf{R}} \sim \eta \tau E_{\mathbf{b}}, \quad \tau \rightarrow 0$,

$q^{\mathbf{R}} \sim \frac{\eta(1-\omega)}{3 \tau} E_{\mathrm{b}}, \quad \tau \rightarrow \infty$.

(For the introduction of $\kappa_{M}$ and $\eta$ into a formulation for arbitrary optical thickness, see Traugott [18, 19], Cogley et al., [20], Gilles et al. [21], Arpaci and Gözüm [22], and Arpaci and Bayazitoğlu [23]).

By inspection, the heat flux based on the assumption of isotropic radiative stress (pressure) and valid for an arbitrary optical thickness may be written as

$q^{\mathbf{R}} \sim \frac{\eta \tau E_{\mathbf{b}}}{1+3 \tau^{2} /(1-\omega)}$

which reduces to thin gas heat flux and the thick gas heat flux as $\tau \rightarrow 0$ and $\tau \rightarrow \infty$, respectively.

Effects of the emission and absorption on the radiation heat flux are shown in Fig. 1. While the effect of emission is monotonic, the effect of absorption diminishes for the thin gas and thick gas limits, and reaches the maximum $q^{\mathbf{R}} / \eta E_{\mathrm{b}}=1 / 2 \sqrt{3}$ at about $\tau=1 / \sqrt{3}$. The effect of scattering, as well as that of absorption, are shown in Fig. 2. Since the scattering implies lost energy, the decreasing $q^{\mathbf{R}} / \eta E_{\mathrm{b}}$ for increasing $\omega$ is expected. In the limit for perfect scattering $\omega=1$ and $q^{\mathrm{R}} / \eta E_{\mathrm{b}}=0$. Next, the effect of radiation on combustion is illustrated by applying the foregoing radiation heat flux to the laminar flame propagation.

\section{LAMINAR FLAME}

Considering a stationary plane deflagration wave of thickness $\delta$, into which a combustible gas mixture flows at velocity $S_{\mathrm{L}}$ [laminar flame speed (Fig. 3)]. Balance of thermal energy for the con. trol volume shown in this figure readily gives

$\rho_{u} S_{L} \Delta h^{0} \sim \Delta q^{K}+\Delta q^{\mathrm{R}}$.

Here $\Delta h^{0}$ is the heat of reaction (energy released per unit mass of the reactant mixture). Hereafter, subscripts $f$ and $u$ will be used for the properties of 


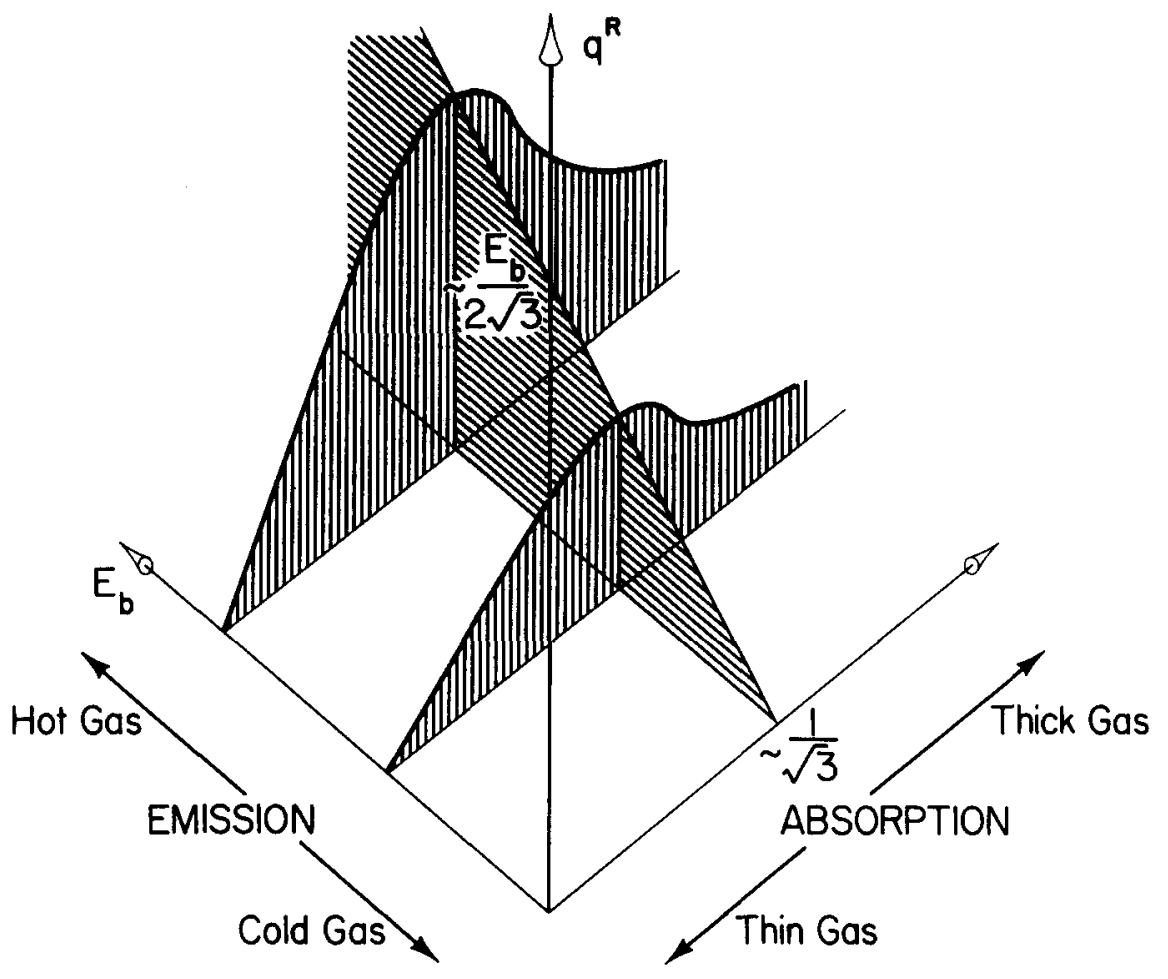

Fig. 1. Effect of emission and adsorption on the radiation heat flux.

flame and unburned gas mixture, respectively, and superscripts $\mathrm{K}$ and $R$ for conduction and radiation. Noting that

$\Delta h^{0}=C_{\mathrm{p}}\left(T_{f}-T_{\mathrm{u}}\right)$

where $C_{\mathrm{p}}$ is an average specific heat of the unburned mixture, and the entire heat release associated with $\Delta h^{0}$ is transferred upstream by conduction

$\Delta q^{\mathrm{K}} \sim \lambda \frac{T_{\mathrm{f}}-T_{\mathrm{u}}}{\delta}$,

and by radiation [to be obtained from Eq. (5)]

$\Delta q^{\mathrm{R}} \sim \frac{\eta \tau}{1+3 \tau^{2} /(1-\omega)}\left(E_{\mathrm{bf}}-E_{\mathrm{bu}}\right)$.

In view of the qualitative nature of this study, linearizing the effect of radiation,

$E_{\mathrm{bf}}-E_{\mathrm{bu}} \sim 4 \sigma T_{M}^{3}\left(T_{\mathrm{f}}-T_{\mathrm{u}}\right)$, where $T_{\mathrm{M}}$ is a characteristic mean temperature, say the adiabatic flame temperature. In terms of Eq. (10), Eq. (9) may be rearranged as,

$\Delta q^{\mathrm{R}} \sim \frac{\eta \tau 4 \sigma T_{M}{ }^{3}}{1+3 \tau^{2} /(1-\omega)}\left(T_{\mathrm{f}}-T_{\mathrm{u}}\right)$.

Inserting Eqs. (7), (8), and (11) into Eq. (6) gives

$\rho_{\mathrm{u}} S_{\mathrm{L}} C_{\mathrm{P}} \sim \frac{\lambda}{\delta}+\frac{\delta \tau}{1+3 \tau^{2} /(1-\omega)} 4 \sigma T_{M}{ }^{3}$

Now, one needs to relate the flame speed to the flame thickness. Noting that the mass of combustible material per unit area and time flowing into the control volume is $\rho_{\mathrm{u}} S_{\mathrm{L}}$, and that flame consumes these reactants at a rate $m \delta, m$ being the reaction rate (mass of the reactant mixture converted per unit volume and time), the conservation of 


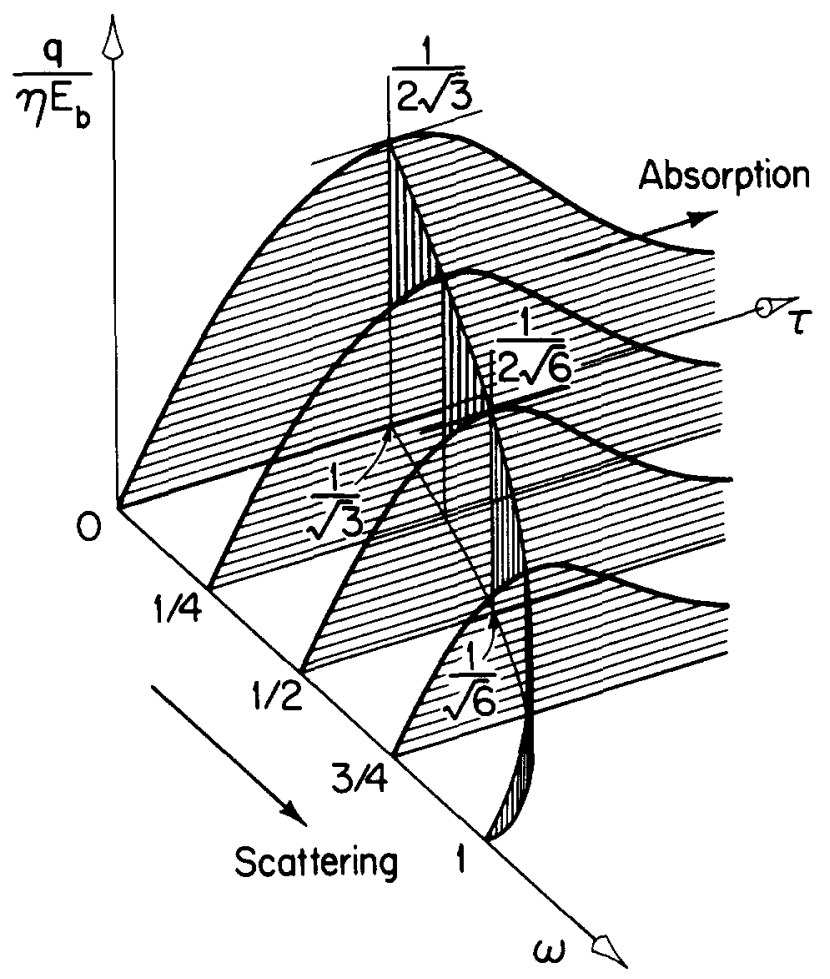

Fig. 2. Effect of scattering and absorption on the radiation heat flux.

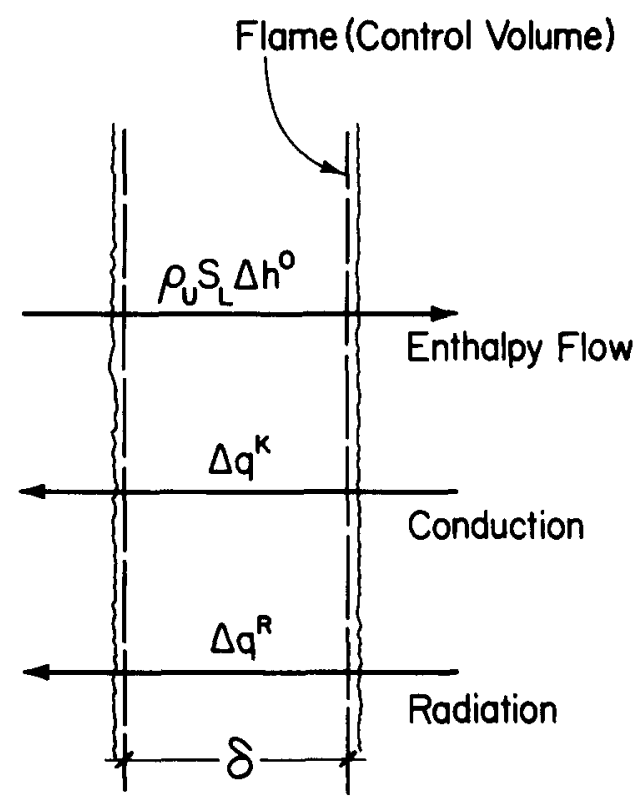

Fig. 3. Energy balance. mass for the control volume yields

$\rho_{\mathrm{u}} S_{\mathrm{L}}=\dot{m} \delta$

Eliminating $S_{\mathrm{L}}$ between Eqs. (12) and (13) results in, after some rearrangement,

$\delta^{2}-\left(\frac{\eta \tau}{1+3 \tau^{2} /(1-\omega)} \frac{4 \sigma T_{M}{ }^{3}}{\dot{m} C_{\mathrm{p}}}\right) \delta-\frac{\lambda}{\dot{m} C_{\mathrm{p}}} \sim 0$,

where the term in parentheses shows the effect of radiation. Employing the Boltzmann number

$B=\frac{4 \sigma T_{M}^{3}}{\dot{m} C_{\mathrm{p}}} \sim \frac{\text { Emission }}{\text { Enthalpy flow }}$

(this number exists in the literature which deals with the dimensionless numbers describing only the diffusion approximation; see, for example, 
Vincenti and Krueger [14] and Penner and Olfe [24]), introducing the radiation number

$R=\frac{\eta \tau B}{1+3 \tau^{2} /(1-\omega)} \sim \frac{\text { Total radiation }}{\text { Enthalpy flow }}$,

Eq. (14) may be rearranged as

$\delta^{2}-R \delta-\left(\lambda / \dot{m} C_{\mathrm{p}}\right) \sim 0$.

In the absence of radiation, $R=0$ and Eq. (17) reduces to

$\delta_{\mathrm{K}}=\left(\lambda / \dot{m} C_{\mathrm{p}}\right)^{\mathbf{1 / 2}}$.

Divide each term of Eq. (17) by $\delta_{\mathrm{K}}{ }^{2}$ and introduce the relative flame thickness

$\Delta=\delta / \delta_{\mathbf{K}}$

hence Eq. (17) may be rearranged as

$\Delta^{2}-H \Delta-1 \sim 0$

where $H$ is the heat transfer number,

$H=\frac{\eta \tau P}{1+3 \tau^{2} /(1-\omega)} \sim \frac{\text { Total radiation }}{\text { Conduction }}$,

and $P$ is the Planck number,

$P=\frac{4 \sigma T_{M}^{3}}{\kappa / \delta_{\mathrm{K}}} \sim \frac{\text { Emission }}{\text { Conduction }}$.

The positive root of Eq. (20) is

$\Delta \sim \frac{1}{2} H+\sqrt{\left(\frac{1}{2} H\right)^{2}+1}$.

For small $H$, employing the binomial theorem, the conduction dominated flame thickness is found to be

$\Delta \sim 1+\frac{1}{2} H+\frac{1}{8} H^{2}$.

For large $H$, rearranging Eq. (23) as

$\Delta \sim \frac{1}{2} H\left(1+\sqrt{1+1 /\left(\frac{1}{2} H\right)^{2}}\right)$, then using the binomial theorem, one gets the radiation-dominated flame thickness,

$\Delta \sim H+\frac{1}{H}$

The behavior of the relative flame thickness characterized by Eq. (23), and its tangent and the asymptote given by Eqs. (24) and (25), are shown in Fig. 4.

At a given level of emission and scattering, the radiation flux is maximum when

$\frac{d}{d \tau}\left(\frac{\tau}{1+3 \tau^{2} /(1-\omega)}\right)=0$,

which yields

$\tau=\left(\frac{1-\omega}{3}\right)^{1 / 2}$

For this value of the optical thickness, the heat transfer number is

$H_{\max }=\eta\left(\frac{1-\omega}{3}\right)^{1 / 2} P$

For $\omega=0$, Eq. (27) gives

$\left(H_{\max }\right)_{\max }=\frac{1}{\sqrt{3}} \eta P$.

Finally, noting from Eq. (13) that

$S_{L} \sim \delta$,

the foregoing development on the film thickness may be extended to the flame speed.

\section{RESULTS AND DISCUSSION}

A start is made in this study to understand qualitatively the mean effect of radiation on the laminar flame propagation. It is found that the radiation increases the flame thickness and the flame speed. Since the contribution of conduction and the ra- 


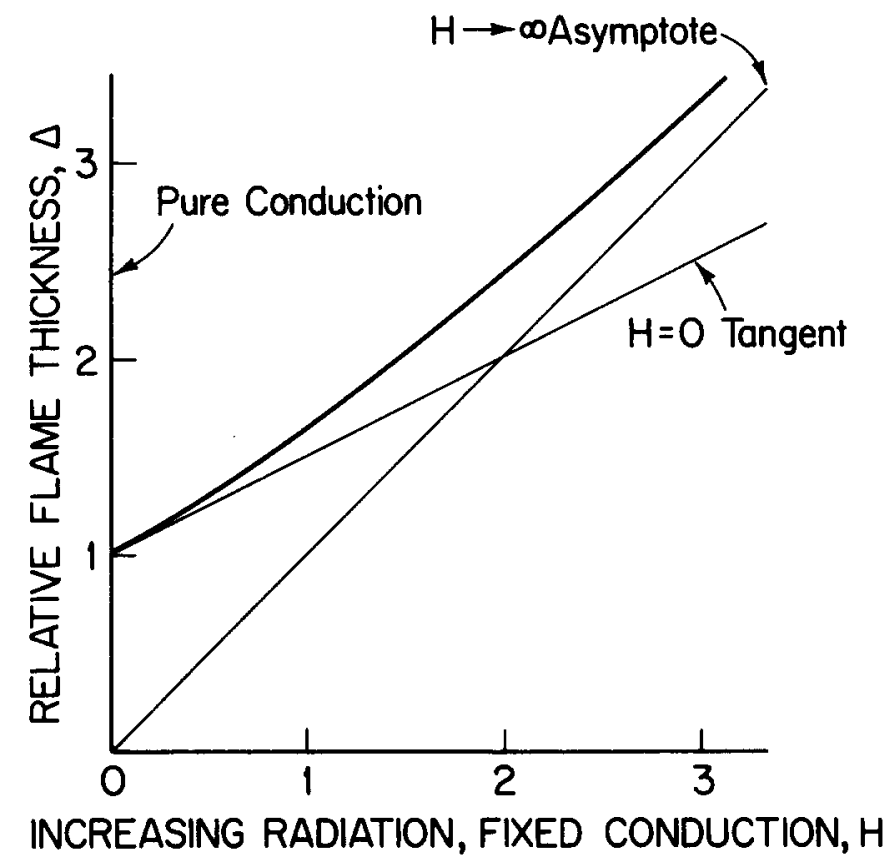

Fig. 4. Effect of radiation of the laminar flame thickness.

diation to the heat flux is cumulative, this result is expected.

The emission, absorption, and scattering of radiation are characterized by the blackbody emissive power, optical thickness, and albedo. A heat transfer number including all effects of radiation relative to conduction,

$H=\frac{\eta \tau P}{1+3 \tau^{2} /(1-\omega)} \sim \frac{\text { Total radiation }}{\text { Conduction }}$,

is introduced. Here $P$ is the Planck number,

$P=\frac{4 \sigma T_{M}^{3}}{\kappa / \delta_{K}} \sim \frac{\text { Emission }}{\text { Conduction }}$.

Assumptions leading to the foregoing explicit form of the heat transfer number are isotropic stress (pressure), isotropic scattering, and the correct mean absorption for the thin gas and thick gas limits.

Clearly, Figs. 1 and 2 illustrate that the effects of emission and scattering are monotonous. Increasing emission or decreasing scattering increases the heat transfer number, which in turn increases the flame thickness and the flame speed. However, the effect of absorption has an extremum. It diminishes for small and large optical thickness, and reaches a maximum at

$\tau=\left(\frac{1-\omega}{3}\right)^{1 / 2}$

At this optical thickness, the heat transfer number has its maximum,

$H_{\max }=\eta\left(\frac{1-\omega}{3}\right)^{1 / 2} P$,

whose relative maximum corresponding to negligible scattering $(\omega=0)$ is

$\left(H_{\max }\right)_{\max }=\frac{1}{\sqrt{3}} \eta P$.

Clearly, the heat transfer number and Planck number are total radiation and emission effects 
relative to conduction. For cases of negligible conduction, another set of dimensionless numbers is needed. Dividing the heat transfer number with the usual Peclet number,

$$
\Pi=\frac{V \delta_{\mathbf{K}}}{a},
$$

one gets the radiation number

$R=\frac{H}{\text { II }}=\frac{\eta \tau B}{1+3 \tau^{2} /(1-\omega)} \sim \frac{\text { Total radiation }}{\text { Enthalpy flow }}$,

where $B$ is the Boltzman number,

$B=\frac{4 \sigma T_{M}^{3}}{\dot{m} C_{\mathrm{p}}} \sim \frac{\text { Emission }}{\text { Enthalpy flow }}$.

Another possibility for dimensionless numbers characterizing the radiation is the inverse of $R$ and $B$, which may be defined as the radiation Peclet number and emission Peclet number:

$\Pi_{\mathrm{R}}=R^{-1}, \quad \Pi_{\mathrm{E}}=B^{-1}$.

An important difference between the conduction related and the radiation related film thicknesses is that

$\delta_{\kappa} \sim(\text { Conduction })^{1 / 2}, \quad \delta_{\mathrm{R}} \sim$ Radiation.

This difference comes from the fact that the conduction is associated with a characteristic length proportional to the flame thickness, while the radiation is associated with a characteristic length related to the optical thickness.

In view of the fact that

$S_{L} \sim \delta$

according to the conservation of mass stated by Eq. (13), the effects of radiation on the flame thickness and the flame velocity are similar.

Because of the qualitative nature of the present investigation, no attempt is made here to evaluate numerically the ranges of the heat transfer number $H$ representative for special areas. Here it may be worth mentioning a number of possible formula- tions whose solutions would show some quantitative effects of radiation. A good starting point may be the theory of Zeldovich. Frank-Kamenetskii, and Semenov, which includes the simultaneous effect of the diffusion of species and that of heat (see Williams [11] or Glassman [12]). Spectral effects of radiation are essential and should now be taken into account. A number of overly simplified models such as the picket-fence (see, for example, Sparrow and Cess [13]) or two-step local or continuous bands (Liu and Clarke [25]) may represent, to a very first order, the quantitative effects of radiation. However, a much more realistic model, which would involve and represent the actual behavior of particles, is needed. These particles, which are the result of incomplete combustion of the fuel, usually play the dominant role in flame and smoke calculations. They give rise to continuous spectral characteristics which contrast the absorption bands of combustion gases. The most recent studies consider only the absorption contribution of the particulates, which is valid when particles (of mean diameter $D$ ) are small relative to the characteristic wavelength $\lambda^{*}$ of radiation, $\pi D \ll \lambda^{*}$. Recent studies $[26,27]$, show that, under typical conditions in flames, the soot may be treated as a gas with negligible scattering properties. Accordingly, the effect of gas radiation on the present problem may be reduced to that of continuous soot absorption and that of discrete absorption of $\mathrm{CO}_{2}$ and $\mathrm{H}_{2} \mathrm{O}$. In the literature, a computational solution for a simpler model assuming a system of suspended carbon particles as an absorbing and nonscattering medium is given by Ozerova and Stepanov [28]. However, for large-scale fires, smoke, and coal furnaces, particulates of larger sizes, $\pi D \gg$ $\lambda^{*}$, are present, and the scattering of radiation becomes quite significant. In this case, the radiation effects include the absorption of gases and the $a b$ sorption and scattering of liquid or solid particles. These particles usually assume temperatures quite different than those of the gas phase. All these complexities suggest the consideration of approximate methods as the viable approach to the solution of the present problem.

Two solution procedures, the two-flux model for all size particles (including appreciable or negli- 
gible scattering) and the linear anisotropic scattering model only for sufficiently large particles including scattering, are available [29]. An approximate analytical study using these solution procedures, as well as including the foregoing quantitative effect of gas radiation, is under progress.

The mathematical structure of the present problem is also suitable to a solution by an asymptotic matching [30]. However, in view of the physical assumptions made, any elaborate solution by a formal procedure appears to be unwarranted.

\section{REFERENCES}

1. Nusselt, W., V.D.I. 68:124, 194 (1924).

2. Sarofim, A. F., and Hottel, H. C., Proc. Sixth Int. Heat Transfer Conf. 1,199 (1978).

3. Smoot, L. D., and Pratt, D. T. (Eds.), Pulverized Coal Combustion and Gasification, Plenum, 1979.

4. Godridge, A. M., and Read, A. W., Progr. Energy Combust. Sci. 2:83 (1976).

5. Smoot, D. L., Eighteenth Symposium (International) on Combustion, Combustion Inst., 1980, p. 43.

6. Krazinski, J. L., Buckius, R. O., and Krier, H., Prog. in Energy Comb. Sci. 5, Pergamon Press, 1979, p. 31.

7. de Ris, J., Seventeenth Symposium (International) on Combustion, Combustion Inst., 1979, p. 1003.

8. Buckius, R. O., and Tien, C. L., Int. J. Heat Mass Transfer 20:93 (1977).

9. Selcuk, N., Siddall, R. G., and Beer, J. M., Sixteenth Symposium (International) on Combustion, Combustion Inst., 1976, p. 53.

10. Landau, L. D., and Lifshitz, Fluid Mechanics, Course of Theoretical Physics, 6, Pergamon Press, Oxford, 1959.

11. Williams, F. A., Combustion Theory, Addison-Wesley, Reading, MA, 1965.
12. Glassman, I., Combustion, Academic Press, New York, 1977.

13. Sparrow, E. M., and Cess, R. D., Radiation Heat Transfer, Hemisphere Publ. Corp., Washington, 1978.

14. Vincenti, W. G., and Kruger, C. H., Introduction to Physical Gas Dynamics, Wiley, New York, 1965.

15. Sampson, D. H., Radiative Contributions to Energy and Momentum in a Gas, Wiley, New York, 1965.

16. Ozisik, M. N., Radiative Transfer, Wiley-Interscience, New York, 1973.

17. Siegel, R., and Howell, J. R., Thermal Radiation Heat Transfer, Hemisphere Publishing Corp., Washington, 1981.

18. Traugott, S. C., in Proc. 1963 Heat Trans. Fluid Mech. Inst. (A. Roshko, B. Sturtevant, and D. R. Bartz, Eds.), Stanford Univ. Press, Stanford, CA, 1963, p. 1.

19. Traugott, S. C., AIAA J. 4:541 (1966).

20. Cogley, A. C., Vincenti, W. G., and Gilles, S. E., AIAA J. 6:661 (1968).

21. Gilles, S. E., Cogley, A. C., and Vincenti, W. G., Int. J. Heat Mass Transfer 12:445 (1969).

22. Arpaci, V. S., and Gözüm, D., Physics of Fluids 16: 581 (1973).

23. Arpaci, V. S., and Bayazitoğlu, Y., Physics of Fluids $16: 589$ (1973).

24. Penner, S. S., and Olfe, D. B., Radiation and Reentry, Academic Press, New York, 1968.

25. Liu, J. T. C., and Clarke, J. H., Physics of Fluids 10 : 2088 (1967).

26. Felske, J. D., and Tien, C. L., Combustion Sci. Tech. 7:25 (1973).

27. Hubbard, G. L., and Tien, C. L., J. Heat Transfer 100:235 (1978).

28. Ozerova, G. E., and Stepanov, A. M., Combustion, Explosion and Shock Waves 9:543 (1973).

29. Tong, T. W., and Tien, C. L., J. Quant. Spectrosc. Radiat. Transfer 24:491 (1980).

30. Ferguson, C. R., Ph.D. dissertation, MIT, 1977.

Received 22 May 1981; revised 8 September 1981 